\title{
OI 24 Efavirenz and rifampicin in the South African context: is there a need to dose increase efavirenz with concurrent rifampicin therapy?
}

\author{
C Orrell ${ }^{* 1}$, K Cohen ${ }^{2}$, P Ive ${ }^{3}$, I Sanne ${ }^{3}$ and R Wood ${ }^{1}$
}

\author{
Address: ${ }^{1}$ Desmond Tutu HIV Centre, UCT, Cape Town, South Africa, ${ }^{2}$ Division of Clinical Pharmacology, UCT, Cape Town, South Africa and \\ 3Wits Health Consortium, Johannesburg, South Africa \\ * Corresponding author
}

from Ninth International Congress on Drug Therapy in HIV Infection

Glasgow, UK. 9-13 November 2008

Published: 10 November 2008

Journal of the International AIDS Society 2008, I I (SuppI I):O9 doi:I0.II86/I758-2652-II-SI-O9

This abstract is available from: http://www.jiasociety.org/content/II/SI/O9

(c) 2008 Orrell et al; licensee BioMed Central Ltd.

\section{Background}

In the developing world many people are co-infected with HIV and tuberculosis (TB). Recommended first-line antiretroviral therapy (ART) commonly includes efavirenz (EFV). Anti-tuberculous therapy includes rifampicin (RFN). There remains debate about whether a dose increase of EFV from the standard dose of $600 \mathrm{mg}$ to an increased dose of $800 \mathrm{mg}$ daily is required with concomitant RFN, which induces cytochrome p450 isoenzymes that may reduce levels of EFV.

\section{Methods}

Individuals from the CIPRA-South Africa adult treatment cohort on EFV-based ART with concomitant TB were dosed with either an increased (800 mg) or standard (600 $\mathrm{mg}$ ) dose of EFV during TB treatment. After TB therapy all were given standard EFV doses. Two mid-dose EFV concentrations were determined from each individual: the first after 4 weeks of concomitant EFV and RFN therapy, and the second at least 4 weeks after TB therapy was completed. Mid-dose EFV concentrations were compared across groups using the Mann-Whitney U-test and within individuals using the Wilcoxon signed rank test.

\section{Summary of results}

Paired samples were collected from 60 individuals; 38 $(63 \%)$ were women. The median weight was $60 \mathrm{~kg}$ (IQR $52-65 \mathrm{~kg}$ ). The median CD4 count at ART baseline was 110 cells/mm3 (IQR 35-158), and median viral load 5.5 $\log$ (IQR 5.1-5.9). Thirty-one (52\%) were taking $800 \mathrm{mg}$ EFV during TB treatment and $29(48 \%)$ had the standard dose. There was no significant difference in the median EFV concentrations on RFN in the $800 \mathrm{mg}$ group [3.2 ug/ $\mathrm{ml}$ (IQR 1.5-6.5)], compared to the $600 \mathrm{mg}$ group [2.85 $\mathrm{ug} / \mathrm{ml}$ (IQR 1.8-5.0)]. The median EFV concentrations off RFN did not differ significantly either [ $800 \mathrm{mg}$ group: 2.15 ug/ml (IQR 1.38-3.09); 600 mg group: $2.4 \mathrm{ug} / \mathrm{ml}$ (IQR 1.4 to 4.3)]. There was no evidence of a difference between concentrations within the individual if on 600 $\mathrm{mg}$ dose at both timepoints nor if on $800 \mathrm{mg}$ dose at first time-point. There was no increase in EFV-linked adverse effects in any group.

\section{Conclusion}

In our population there is no reduction in mid-dose EFV concentrations with concomitant RFN therapy. The pharmacogenetic profile cytochrome $\mathrm{p} 450$ isoenzyme, particularly the 2B6 isoenzyme, in this South African population may differ from the other populations where the majority of pharmacokinetic work has been completed. It was not necessary to increase the dose of EFV during concomitant TB therapy in this South African population. 